\title{
Targeted designed variants of alpha-2- macroglobulin (A2M) attenuate cartilage degeneration in a rat model of osteoarthritis induced by anterior cruciate ligament transection
}

\author{
Yang Zhang ${ }^{1}$, Xiaochun Wei ${ }^{1}$, Shawn Browning ${ }^{2}$, Gaetano Scuderi ${ }^{2}$, Lewis S. Hanna ${ }^{2}$ and Lei Wei ${ }^{1,3^{*}}$
}

\begin{abstract}
Background: The study was performed to evaluate whether targeted alpha-2-macroglobulin (A2M) variants have a similar or enhanced function at wild-type (wt)-A2M to attenuate cartilage degeneration in vivo.

Methods: In and ex-vivo experiment, bovine cartilage explants (BCE) were incubated with TNF-a and IL-1 $\beta$ with or without wt-A2M or A2M variants. Cartilage catabolism was measured in culture supernatant by sulfated glycosaminoglycan (sGAG). In an in-vivo experiment, 2-month-old male Wistar rats $(n=77)$ were randomly divided into seven groups and treated with different doses of A2M or its variants by intra-articular injection at 24 hours and day 14 after anterior cruciate ligament transection (ACLT), receiving (1) ACLT/PBS; (2) ACLT/wt-A2M (0.153 mg); (3) ACLT/CYT-108 A2M (0.153 mg); (4) ACLT/CYT-108 A2M (0.077 mg); (5) ACLT/CYT-98 A2M (0.153 mg); (6) ACLT/CYT98 A2M $(0.077 \mathrm{mg})$; or (7) sham/PBS. The joints and synovial lavage were collected 8 weeks after surgery. Fluorescence molecular tomography was used to monitor inflammation in vivo using probes ProSense and MMPSense at 24 hours, and weeks 2, 4, and 6 after surgery. The cartilage damage was quantified using Osteoarthritis Research Society International score and matrix metalloproteinase (MMP)-3, -13, collagen (Col) X, Col 2, Runx2, and aggrecan (Acan) were detected by immunohistochemical analysis (IHC), ELISA, and RT-PCR.
\end{abstract}

Results: A2M variants inhibited catabolism in the BCE model by up to 200\% compared with wt-A2M. ProSense and MMPSense were dramatically increased in all groups after surgery. Supplemental A2M or its variants reduced ProSense and MMPSense compared with the PBS treatment. Less cartilage damage, lower MMP-13 and Col 2 degraded product, and stronger $\mathrm{Col} 2$ synthesis were detected in animals treated with A2M or its variants compared with PBS-treated animals. A2M and its variants enhanced Col 2 and Acan synthesis, and suppressed MMP-3, MMP-13, Runx2, and Col X production. A2M-108 variant demonstrated less cartilage damage compared with wt-A2M and A2M-98 variant.

Conclusion: The targeted variants of A2M have a chondroprotective effect similar to wt-A2M. However, A2M-108 variant has enhanced function to attenuate cartilage degeneration compared with wt-A2M.

Keywords: Targeted designed variants of A2M, PTOA, Rat ACLT OA model

\footnotetext{
* Correspondence: Lei_Wei@brown.edu

'Department of Orthopedics, the second hospital of the Shanxi Medical University, Taiyuan, China

${ }^{3}$ Department of Orthopedics, Alpert Medical School of Brown University/

Rhode Island Hospital, Providence, RI, USA

Full list of author information is available at the end of the article
} 


\section{Background}

Although osteoarthritis (OA) affects over 40 million Americans, its pathogenesis remains undefined. OA progression is due, at least in part, to the upregulation of inflammation mediators and proteases [1-4]. Since collected evidence has demonstrated that elevated levels of catabolic enzymes in synovial fluid (SF) induce chondrocyte death and cartilage matrix degeneration within one week of joint injury [5-9], early intervention strategies should focus on reducing these cartilage-degrading proteases within a similar time frame. Previous studies have indicated that catabolic proteases and cytokines reach their peak within $48 \mathrm{~h}$ after joint injury, which initiates cell death and cartilage matrix degeneration [1]. Thus, early intervention to reduce these catabolic proteases and cytokines is critical to prevent or delay cartilage degeneration.

Evidence from our group [4, 10-12] and others [1, 13] suggests that new molecular interventions targeting these catabolic enzymes can potentially arrest these adverse events and preserve joint health. It is unlikely, however, that blocking only one of these catabolic factors would be enough to repress the multi-catabolic inflammation factors after joint injury.

Our laboratory and others have demonstrated that serum alpha-2-macroglobulin (A2M) is a promising bioinhibitor for most of these catabolic enzymes, though it is not adequately present in the joint due to the large molecular weight of A2M, which prevents it from migrating into the SF [5, 14-16]. Recently, we have shown that supplemental intra-articular injection of A2M shortly after joint injury provides chondral protection in anterior cruciate ligament (ACL) injury of the knee by reducing these catabolic enzymes [5]. However, administering serum A2M from patients is time-consuming and complex, which may limit clinical application. We designed targeted variants of $\mathrm{A} 2 \mathrm{M}$ to enhance $\mathrm{A} 2 \mathrm{M}$ inhibitory function to overcome the purification/concentration burden associated with utilizing circulatory wild-type A2M.

Cytonics scientists have synthesized more than 100 variants and tested their inhibition against 12 proteases mostly implicated in cartilage digestion. The two variants that demonstrated the highest inhibitory characteristics toward largest number of proteases were selected; these were variants 98 and 108 (data not shown). In this study, we used bovine articular cartilage explants (BCE) to screen targeted designed A2M variants in vitro, fluorescence molecular tomography (FMT), a new advanced method for measuring certain catabolic protease levels in vivo, histological and immunohistochemical analyses, and RT-PCR, to compare the efficacy of wild-type-A2M (wt$\mathrm{A} 2 \mathrm{M}$ ) and two A2M variants in vivo using our established rat anterior cruciate ligament transection (ACLT) OA model. We have clearly shown that cartilage-degrading proteases, such as elastase, Cathepsin G, B, L and S, and matrix metalloproteinase (MMP)-3, MMP-9, and MMP13, and ADAMTS 4 and 5 are potently inhibited by A2M and its targeted variants in both the BCE model and a surgically induced OA rat model. Supplemental A2M and its variants attenuate cartilage degeneration and inhibit MMP-13 compared with rats treated by PBS. Our evidence suggests that these molecular variants of A2M, especially 108 variant, have a potentially enhanced function relative to wt-A2M to block multiple catabolic proteases and attenuate OA development in the rat ACLT-OA model.

\section{Methods}

\section{BCE model and treatment}

BCE were isolated from heifers 1.0-1.5 years old and were equilibrated for 3 days in culture medium. To degrade cartilage by cytokine treatment, BCE are incubated for 3 days in DMEM containing 10\% fetal bovine serum with or without TNF- $\alpha 80 \mathrm{ng} / \mathrm{ml}$ and IL-1 $\beta 8 \mathrm{ng} / \mathrm{ml}$. Cartilage degradation is inhibited with the addition of either a twofold serial dilution standard curve of wt-A2M (2.0$0.13 \mathrm{mg} / \mathrm{ml})$ or $\mathrm{A} 2 \mathrm{M}$ variants $(0.25 \mathrm{mg} / \mathrm{ml})$ designed to potently inhibit OA. Cartilage catabolism is measured in culture supernatant by sulfated glycosaminoglycan (sGAG) compared to a standard curve of chondroitin sulfate using di-methylmethylamine staining $[4,11,17]$.

The bait region of A2M variants was optimized by placing specific sequences around the cleavage sites from the native substrates for ADAMTS 5 and 4, MMPs, elastase and cathepsin. The difference between variables was mainly the order of these sequences. The size of the bait region was the same as the wt and care was taken that the variant sequences maintained a random coil structure similar to the wt sequence (NCBI Reference Sequence: NM_000014.4). Hek293 cells were transiently transfected with plasmid containing the sequence for the new A2M using the Transit Pro Transfection Reagent (Mirus, MIR5700) according to manufacturer's protocols. Following incubation the conditioned medium was harvested and centrifuged first at $150 \mathrm{rcf}$ for 5 minutes then at $5850 \mathrm{rcf}$ for 20 minutes, to remove cells and debris. High-performance liquid chromatography (HPLC) and ion exchange chromatography were used for initial purification steps. The eluted A2M from the ion exchange column was further purified using cobalt affinity chromatography. The purified A2M was diluted with PBS.

\section{Rat ACLT OA model and treatment with supplemental intra-articularA2M or injection of its variants}

Two-month-old male Wistar rats $(n=77)$ were purchased from Charles River and randomly divided into seven groups ( $n=11 /$ per group) and treated with different doses of A2M and its variants (provided by Cytonics 
Corp.): (1) ACLT + PBS; (2) ACLT + wt-A2M (0.153 mg); (3) ACLT + CYT-108 A2M (0.153 mg); (4) ACLT + CYT108 A2M (0.077 mg); (5) ACLT + CYT-98 A2M (0.153 mg); (6) ACLT + CYT-98 A2M (0.077 mg); or (7) sham + PBS. ACLT (groups 1-6) or sham surgery (group 7) were performed on the left knee and the right knee served as an internal control, as published previously $[18,19]$. A2M and its variants dissolved in $20 \mu \mathrm{l}$ of PBS were intra-articularly injected $24 \mathrm{~h}$ and 14 days after ACLT. Animals in groups 1 and 7 received an equivalent volume of PBS at identical time points to the experimental groups (2-6) in their left knees to control for any procedural effects. Previous research has demonstrated that there is no statistical difference between ACLT + PBS injection and ACLT without PBS injection in the rat ACLT model. Therefore, we did not compare ACLT + PBS treatment with ACLT without PBS injection in this study. All animals were euthanized at week 8 after the operation.

\section{Monitoring inflammation dynamically in vivo}

FMT is a new, advanced, sensitive, non-ionizing radiation method used to monitor certain catabolic proteases level in real time in vivo [12]. A mix of fluorescent imaging probes ProSense $(10 \mu \mathrm{l}, 13.3 \mu \mathrm{M})$ and MMPSense $(10 \mu \mathrm{l}, 13.3 \mu \mathrm{M})$ were injected intraarticularly $24 \mathrm{~h}$ after each A2M injection. The fluorescent imaging probes were also injected at weeks 4 and week 6 after surgery on both the right (control) and left knees, respectively. FMT was used to monitor the levels of inflammation in vivo $24 \mathrm{~h}$ after injection of ProSense 750 for the detection of plasmin, cathepsin B, L, and S, and MMPSense 680 was used for the detection of MMP-3, MMP-9, and MMP-13. The picomolar concentrations of probes in the knee joint were determined using region of interest analysis. Data are reported as mean $\pm \mathrm{SE}$, with 11 animals per group.

\section{Histologic assessment}

The proximal tibiae were removed from the harvested joints and immersed in $10 \%$ formalin for $72 \mathrm{~h}$. The specimens were decalcified in $20 \%$ ethylenediaminetetraacetic acid solution ( $\mathrm{pH}$ 7.2). Frontal sectioning was performed. The tibial plateau was cut into two approximately equal pieces, an anterior and a posterior one, along the medial collateral ligament in the frontal plane. The two resulting tissue pieces (anterior and posterior half) were then both embedded in a single paraffin block with the cut planes facing down. The blocks were trimmed to expose the cartilage. Ten adjacent sections were collected at intervals of $0 \mu \mathrm{m}, 200 \mu \mathrm{m}$, and $400 \mu \mathrm{m}$. Two serial $6-\mu \mathrm{m}$-thick sections from each interval were stained with Safranin O. Cartilage degradation was quantified using the Osteoarthritis Research Society
International (OARSI) grading system [20]. Three independent observers scored each section blinded, and the scores from the tibial plateau sections were averaged for each individual animal before comparing groups. Our previous study has demonstrated that A2M attenuates cartilage degeneration $[5,17]$. In this study, our prespecified primary outcome measure was to repeat the comparison between the wt-A2M with PBS and then test whether these targeted designed A2M variants can achieve a similar result or even better result than wt using the microscopic OARSI score system [20].

\section{Immunohistochemical analysis}

To detect the distribution of MMP-13, Col 2, Col 2 breakdown product, and Col $\mathrm{X}$ in cartilage, $6-\mu \mathrm{m}$ sections were collected on positively charged glass slides (Thermo Fisher Scientific, Asheville, NC, USA). The sections were dried on a hotplate to increase adherence to the slides. Immunohistochemical (IHC) analysis was carried out using the 3,3'-diaminobenzidine (DAB) streptavidin-peroxidase (SP) DAB Histostain-SP immunohistochemistry kit (ZYMED Laboratories/Invitrogen, Carlsbad, CA, USA). Sections were deparaffinized and rehydrated using conventional methods. Endogenous peroxidase was blocked by treating the sections with $3 \%$ hydrogen peroxide in methanol (Sigma-Aldrich) for 30 minutes. The sections were digested by $5 \mathrm{mg} / \mathrm{ml}$ hyaluronidase in PBS (Sigma-Aldrich) for 20 minutes. The sections were incubated with specific antibodies against MMP-13 (1:100) (Santa Cruz Biotechnology), types II (1:10) (Developmental Studies Hybridoma Bank, University of Iowa, Iowa City, IA, USA), Col X (1:50) (EMD Biosciences, Billerica, MA, USA) and Col 2 breakdown product (1:100) (IBEX Technologies, Mont-Royal, QC, Canada), respectively, at $4{ }^{\circ} \mathrm{C}$ overnight. The negative control sections were incubated with isotype-matched control serum $(2 \mu \mathrm{g} / \mathrm{ml})$ (R\&D Systems, Minneapolis, MN, USA) in PBS. Thereafter the sections were treated sequentially with biotinylated secondary antibody and SP conjugate (ZYMED Laboratories/Invitrogen), then developed in DAB chromogen (ZYMED Laboratories/Invitrogen). The sections were counterstained with hematoxylin (ZYMED Laboratories/Invitrogen). Photomicrographs were taken with a Nikon E800 microscope (Nikon, Melville, NY, USA) [17].

\section{Rat SF lavage collection and analyses}

SF lavages were collected from the knees immediately after euthanasia [4]: $100 \mu \mathrm{l}$ of isotonic saline solution was injected intra-articularly using a 30-gauge insulin syringe inserted through the inferior patellar tendon [2]. With injection the joint capsule was visibly distended. The knee was then manually cycled through flexion and extension 10 times to distribute the fluid within the joint 
before collection by joint aspiration. About half of the fluid that was injected was recovered. The SF was centrifuged at $2000 \mathrm{~g}$ for 10 minutes to remove cells and debris, and frozen at $-80{ }^{\circ} \mathrm{C}$ until analysis. MMP-13 was measured in the SF lavage samples by ELISA following the manufacturer's instructions (Rat MMP13 ELISA Kit Catalog NO.LS-F5518, LifeSpan Biosciences, Inc, WA, USA). Briefly, $100 \mu \mathrm{l}$ of the standard or the sample were added per well and incubated for $2 \mathrm{~h}$ at room temperature (RT). The liquid of each well was aspirated. Then, $100 \mu$ of detection reagent A working solution was added to each well and incubated for $1 \mathrm{~h}$ at $37^{\circ} \mathrm{C}$. After washing, $100 \mu \mathrm{l}$ of detection reagent B working solution was added to each well and incubated for $1 \mathrm{~h}$ at $37{ }^{\circ} \mathrm{C}$. Then, $90 \mu \mathrm{l}$ of substrate solution was added to each well for 30 minutes at $37{ }^{\circ} \mathrm{C}$ (protected from light) after washing 5 times. Finally, $50 \mu \mathrm{l}$ of stop solution was added to each well, and the colorimetric density of the developed plates was determined within 30 minutes using a microplate reader set to $450 \mathrm{~nm}$ (Spectramax M2 ${ }^{\mathrm{e}}$ Multi-Mode Microplate Reader, Molecular Devices, Sunnyvale, CA, USA). The ELISA was performed in duplicate.

\section{Real-time PCR ( $q P C R$ )}

The femoral condyle cartilage was dissected under dissection microscopy. Three rat cartilage samples were pooled ( $\mathrm{n}=9$ per group). Total RNA was isolated using RNeasy isolation kit (Cat. No. 74104, Qiagen, Valencia, CA, USA) [10]; $1 \mu \mathrm{g}$ of total RNA was transcribed into complementary DNA (cDNA) using the iScript ${ }^{\mathrm{TM}}$ cDNA synthesis Kit (Bio-Rad, Hercules, CA, USA). Of the resulting cDNA, $40 \mathrm{ng} / \mathrm{ul}$ was used as the template to quantify the relative content of messenger RNA (mRNA) using QuantiTect SYBR Green PCR kit (QIAGEN, Valencia, CA, USA) with the CFX384 Real-Time PCR Detection System (Bio-Rad Laboratories, Hercules, CA, USA). We used rat Col2a1 forward primer - AAG GGA CAC CGA GGT TTC ACT GG, rat Col2a1 reverse primer - GGG CCT GTT TCT CCT GAG CGT; rat Acan forward primer - CAG TGC GAT GCA GGC TGG CT, rat Acan reverse primer - CCT CCG GCA CTC GTT GGC TG; rat Col10a1 forward primer - CCA GGT GTC CCA GGA TTC CC, rat Col10a1 reverse primer - CAA GCG GCA TCC CAG AAA GC; rat Mmp3 forward primer - TTG TCC TTC GAT GCA GTC AG, rat Mmp3 -3 reverse primer - AGA CGG CCA AAA TGA AGA GA; rat Mmp13 forward primer - GGA CCT TCT GGT CTT CTG GC, rat Mmp13 reverse primer - GGA TGC TTA GGG TTG GGG TC; rat Runx2 forward primer CCGCAC GAC AAC CGC ACC AT, rat Runx2 reverse primer - CGC TCC GGC CCA CAA ATC TC; 18S RNA forward primer - CGG CTA CCA CAT CCA AGG AA,
18S RNA reverse primer - GCT GGA ATT ACC GCG GCT. Relative transcript levels were calculated as

$$
\mathrm{x}=2^{-\Delta \Delta \mathrm{Ct}}
$$

in which $\Delta \Delta \mathrm{Ct}=\Delta \mathrm{Ct} \mathrm{E}-\Delta \mathrm{Ct} \mathrm{C}$, and $\Delta \mathrm{Ct} \mathrm{E}=\mathrm{Ctexp}-\mathrm{Ct} 18 \mathrm{~S}$, and $\Delta \mathrm{Ct} \mathrm{C}=\mathrm{CtC}-\mathrm{Ct} 18 \mathrm{~S}$ as previously described $[5,17]$.

\section{Statistical analysis}

One-way analysis of variance (ANOVA) was used to analyze the differences among mean cartilage damage scoreS, synovial hyperplasia scoreS, FMT scan results, MMP-13 levels, and the mRNA levels of Acan, MMP-3, MMP-13, Runx2, Col2a1, and Col X. The least significant difference (LSD) multiple comparisons test was used to perform pairwise comparisons following the ANOVA. Differences were considered statistically significant at $P<0.05$. Statistical analyses were performed using SPSS 13.0 software.

\section{Results \\ BCE}

The data obtained from BCE culture supernatant demonstrated that wt-A2M (left) and A2M variants CYT-98 and CYT-108 (right) inhibit cartilage catabolism induced by TNF $\alpha$ and IL-1 $\beta$. The absolute sGAG values were $313.5 \mu \mathrm{g} / \mathrm{ml}$ in the untreated cytokine-stimulated explants, $78.6 \mu \mathrm{g} / \mathrm{ml}$ in the A2M-treated cytokine-stimulated explants, $44.6 \mu \mathrm{g} / \mathrm{ml}$ in the CYT-98 A2M-treated cytokinestimulated explants, $37.8 \mu \mathrm{g} / \mathrm{ml}$ in the CYT-108-treated cytokine-stimulated explants, and $183.1 \mu \mathrm{g} / \mathrm{ml}$ in the untreated un-stimulated explants, respectively. The soluble GAG levels determined after treatment with wt-A2M were 1.76-fold and 2.08-fold the levels determined after treatment with variant CYT-98 and CYT-108, respectively. The results also demonstrated that $\mathrm{A} 2 \mathrm{M}$ variants were more effective in inhibiting cartilage catabolism in the BCE model by up to $200 \%$ compared with wt-A2M (Fig. 1 , right) $(P<$ $0.05)$. The variant 108 was the most efficacious inhibitor in the BCE model.

\section{FMT}

Using in vivo deep-tissue imaging methods, real-time information was gained about MMPs and cathepsin biological processes using probes. The levels of ProSense and MMPSense were dramatically increased in all groups after ACLT or sham surgery. MMPs and cathepsin levels peak 2 days after knee joint injury. The highest levels of MMPs (A and B) and cathepsin (C and D), as detected by FMT after ACLT, were observed 2 days after surgery, indicating an early catabolic response. Supplemental A2M or its variants reduced the level of MMPSense (A and B) and ProSense (C and D) compared with the PBS-treated group (Fig. 2, red line) and 


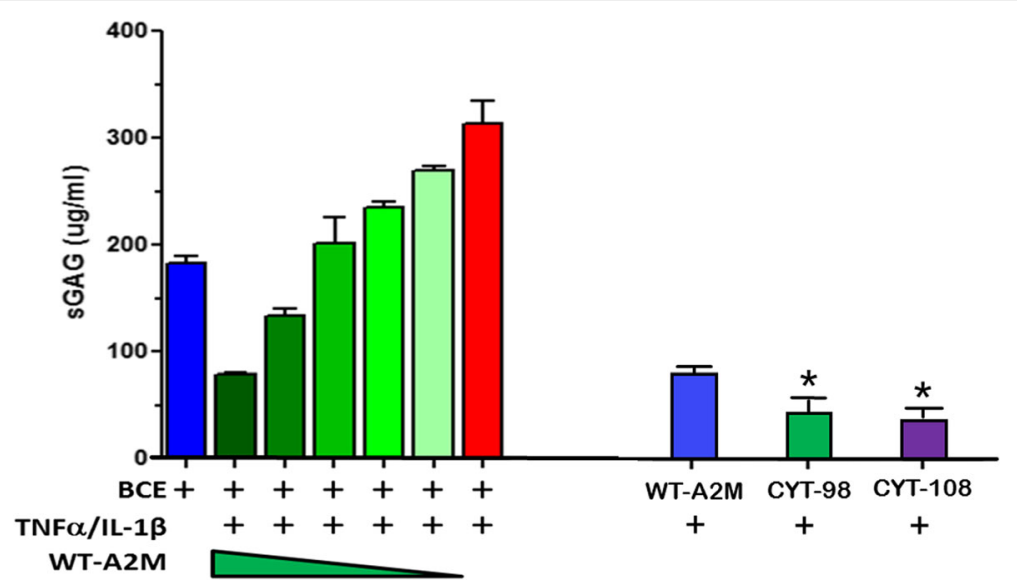

Fig. 1 Wild-type alpha-2-macroglobulin (WT-A2M) (left) and A2M variants CYT-98 and CYT-108 (right) inhibit cartilage catabolism induced by TNFa and IL-1 $\beta .{ }^{*}$ Compared with wt-A2M, $P<0.05$. BCE, bovine articular cartilage explants, SGAG sulfated glycosaminoglycan

the sham group (Fig. 2, blue line). This is consistent with our BCE model data. The ProSense remained at a low level at weeks 4 and 6 after surgery even without treatment. Interestingly, at weeks 4 and 6 without treatment, the level of MMPSense was increased again compared with the sham group, but was lower than in the PBStreated animals. The mean SD region of interest (ROI) signal intensities ( $\mathrm{n}=11$ per group) at each time point over a 6-week period are shown at the bottom (Fig. $2 \mathrm{~b}$ and d: "compared with Sham, $P<0.05 .{ }^{\#}$ compared with PBS, $P<0.05)$.

\section{Supplemental intra-articular injection of wt-A2M and its variants attenuates the severity of $O A$ cartilage degeneration}

Histological analysis (OARSI score) showed that the animals treated with $\mathrm{A} 2 \mathrm{M}$ and its variants had a significant decrease in OA cartilage damage compared with the rats that underwent ACLT and PBS treatment (Fig. 3). Strong Safranin O staining, more cellularity but less chondrocyte cloning, and less fibrillation were observed in the animals treated with A2M and its variants at either concentration compared with the animals treated with the PBS (Fig. 3a). Cartilage in the rats that were treated with A2M variant 108 at high concentration had stronger staining and more intact surface than cartilage in the rats that were treated with variant 108 at low concentration and others, but had weaker staining than the control rats that underwent sham operation. The OARSI grading score indicated that cartilage damage was most severe in rats that underwent ACLT and PBS treatment and the cartilage in rats that underwent sham operation had the least damage $(9.75 \pm 0.88$ and $0.78 \pm 0.11$, respectively; $P<0.05$ ), whereas cartilage damage in the $\mathrm{A} 2 \mathrm{M}$ and the A2M-variants-treated group was significantly less than in the PBS group (wt-A2M: $4.43 \pm 0.52$;
CYT-98-low: $3.36 \pm 0.34 ; \quad$ CYT-98-high: $3.27 \pm 0.32$; CYT-108-low: $3.26 \pm 0.21$; CYT-108-high: $1.98 \pm 0.07$; respectively; $P<0.01$ ) (Fig. $3 b)$. Values are mean \pm SD.

The animals treated with wt-A2M and its variants had reduced synovial hyperplasia

Using hematoxylin/eosin staining, we also observed changes in the synovial membrane in the animals treated with $\mathrm{A} 2 \mathrm{M}$ and its variants compared with the animals treated with PBS (Fig. 4a). Only one or two layers of synovial membrane existed in the sham animals. Synovial hyperplasia was seen in the PBS-treated animals with the thicker synovial membranes, whereas the animals treated with $\mathrm{A} 2 \mathrm{M}$ and its variants had thinner synovial membranes compared with the PBS treated animals. Semi-quantified data are shown in Fig. 4b.

\section{IHC analyses}

IHC staining showed that matrix metalloproteinase 13 (MMP-13) (Fig. 5a), type X collagen (Fig. 5b), and type II degraded products staining (Fig. 5c) were significantly elevated in rats that underwent ACLT and PBS treatment, but were lower in the A2M-treated and A2Mvariants-treated and sham-operated rats, which is consistent with reduced OA damage in these rats (Fig. 3). In contrast, type II collagen expression in articular cartilage was higher in the A2M and A2M-variants-treated and sham-operated rats than in rats that underwent ACLT and PBS treatment (Fig. 5d). The bottom panels of Fig. 3 are higher-magnification views of the boxed areas in the top panels. ELISA further confirmed that A2M and its variants partially reduce the concentration of MMP-13 (Fig. 5e). In $\mathrm{A} 2 \mathrm{M}$ and $\mathrm{A} 2 \mathrm{M}$-ariants-treated rats, the concentration of MMP-13 in SF was significantly lower (A2M: $1.92 \pm 0.32 \mathrm{ng} / \mathrm{ml}$; CYT-98 low: $1.49 \pm 0.33 \mathrm{ng} / \mathrm{ml}$; CYT-98 high: $1.32 \pm 0.17 \mathrm{ng} / \mathrm{ml}$; CYT-108 low: $1.49 \pm$ 

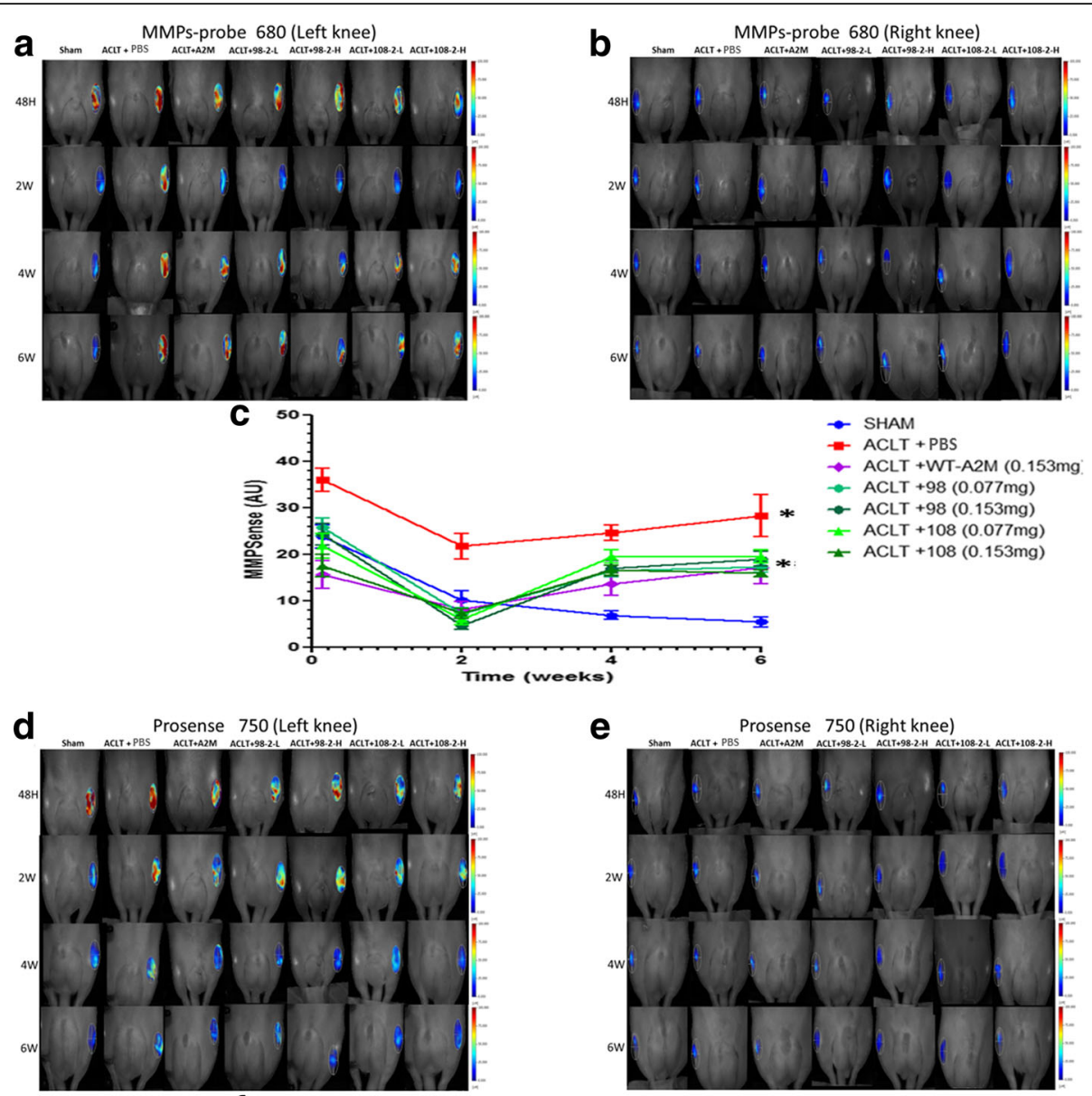

e Prosense 750 (Right knee)
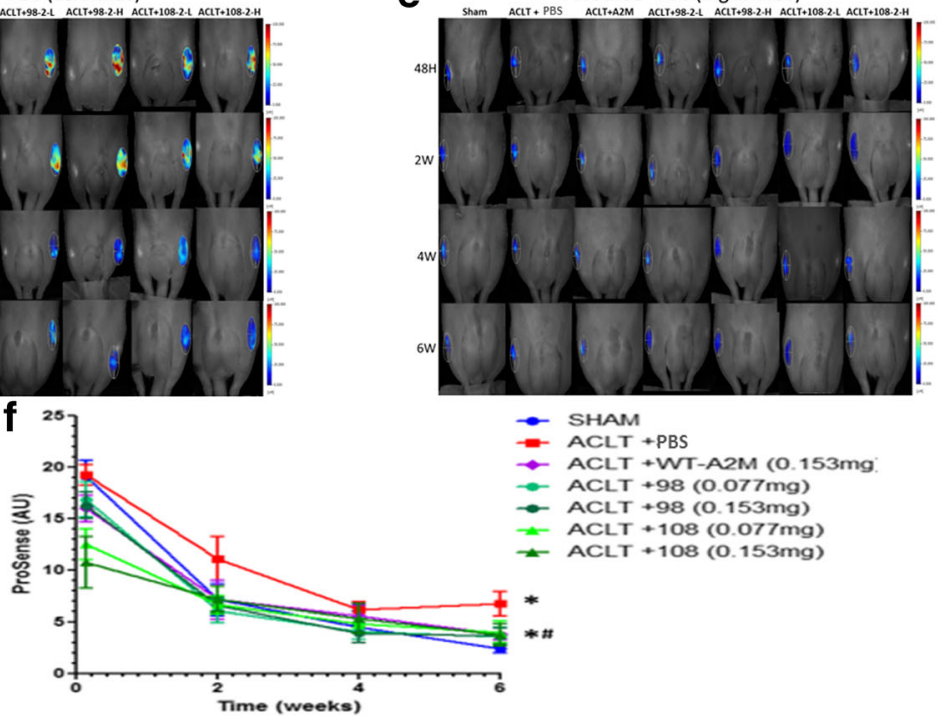

- SHAM

- ACLT +WVT-A2M (0.153mg:

- ACLT +98 (0.077mg)

- ACLT +98 (0.153mg)

- ACLT $+108(0.077 \mathrm{mg})$

$A C L T+108(0.153 \mathrm{mg})$

Fig. 2 Wild-type alpha-2-macroglobulin (WT-A2M) and its variants CYT-98 and CYT-108 inhibit matrix. metalloproteinases (MMPs) (a and b) and cathepsin (d and $\mathbf{e}$ ). MMPs and cathepsin levels peak 2 days after knee joint injury. The levels of MMPs (a) and cathepsin (d), as detected by fluorescence molecular tomography after anterior cruciate ligament transection (ACLT), peaked 2 days after surgery, indicating an early catabolic response that subsided thereafter. The mean SD region of interest signal intensities ( $n=11$ per group) at each time point over a 6-week period are shown (bottom) (c and $\mathbf{f}$ ). ${ }^{*}$ Compared with sham, $P<0.05 .{ }^{*}$ Compared with $\mathrm{PBS}, P<0.05$

$0.23 \mathrm{ng} / \mathrm{ml}$; CYT-108 high: $1.13 \pm 0.10 \mathrm{ng} / \mathrm{ml})$ than that in the rats that underwent ACLT and PBS treatment $(3.26 \pm 0.40 \mathrm{ng} / \mathrm{ml})$ but it was still higher than that in sham-operated rats $(0.92 \pm 0.09 \mathrm{ng} / \mathrm{ml})(P<0.05)$. Values are the mean $\pm \mathrm{SD}$.

Real-time PCR data indicated that supplemental intraarticular injection of wt-A2M and its variants CYT-98 and CYT-108 reduced cartilage matrix catabolism and enhanced anabolic metabolism in the ACLT rat OA model (Fig. 6). The mRNA levels of the MMP-3, MMP13, Runx2, and Col X were expressed at a lower level in rats that were administered $\mathrm{A} 2 \mathrm{M}$ and its variants as compared to rats that underwent ACLT and PBS treatment. In contrast, the levels of mRNA for Col X and aggrecan followed the opposite pattern. Both of them were increased in rats that were administered $\mathrm{A} 2 \mathrm{M}$ and its variants as compared to rats that underwent ACLT and PBS treatment, suggesting that A2M has a positive impact on cartilage matrix anabolism. Values are the mean \pm SEM. \# $P<0.05$ compared with ACLT + PBS; * $P<0.05$ compared with sham; \&Compared with ACLT + A2M, P<0.05, € compared with ACLT + 98- 


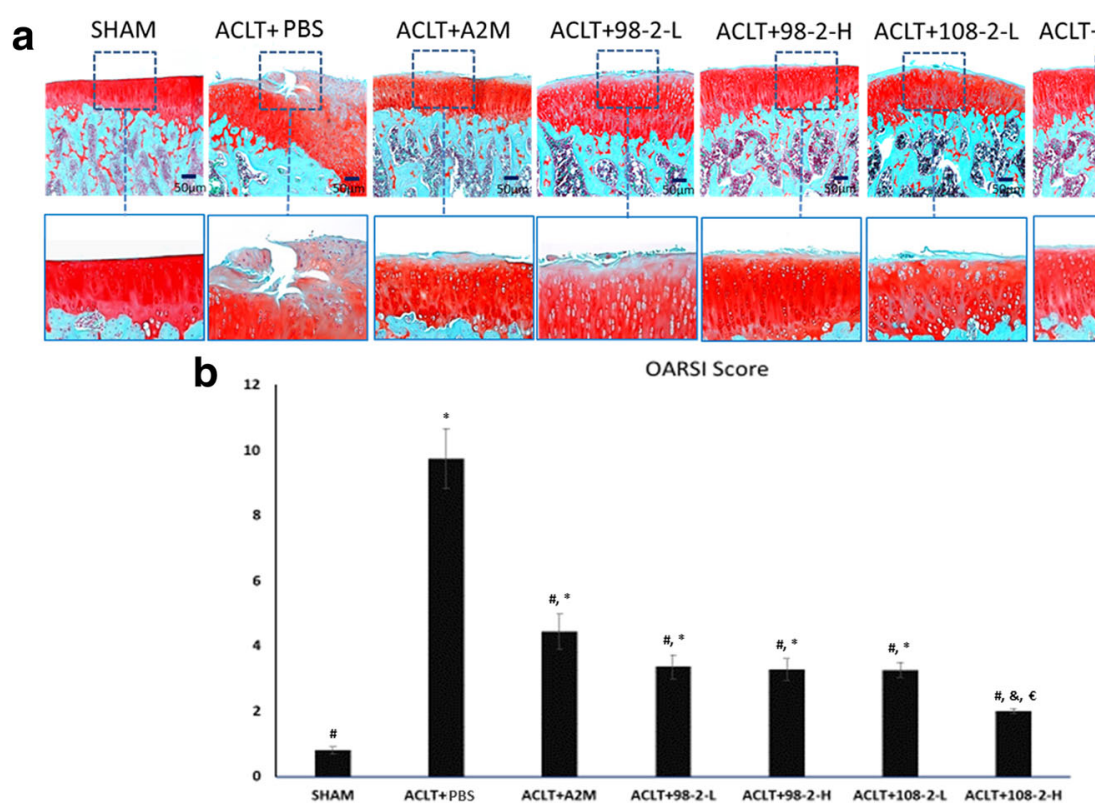

Fig. 3 Supplemental intra-articular injection of wild-type alpha-2-macroglobulin (wt-A2M) and its variants CYT-98 and CYT-108 attenuates cartilage degeneration. a Strong Safranin O staining and a relatively smoother surface were detected in the articular cartilage from the animals treated with wt-A2M and its variants as compared to PBS-treated controls. b The Osteoarthritis Research Society International grading score indicated that cartilage damage was most severe in rats that underwent anterior cruciate ligament transection (ACLT) and PBS treatment, while cartilage in rats that underwent sham operation had the least damage. Cartilage damage was further reduced in the rats that received the high dose of the variants of $A 2 \mathrm{M}$ as compared to the rats that received the low dose of the variants of $\mathrm{A} 2 \mathrm{M}$. Values are the mean $\pm \mathrm{SD}$. ${ }^{\#}$ Compared with $\mathrm{ACLT}+\mathrm{PBS}, P<0.05$; ${ }^{*}$ compared with sham, $P<0.05 ;{ }^{\epsilon}$ compared with $\mathrm{ACLT}+\mathrm{A} 2 \mathrm{M}, P<0.05$

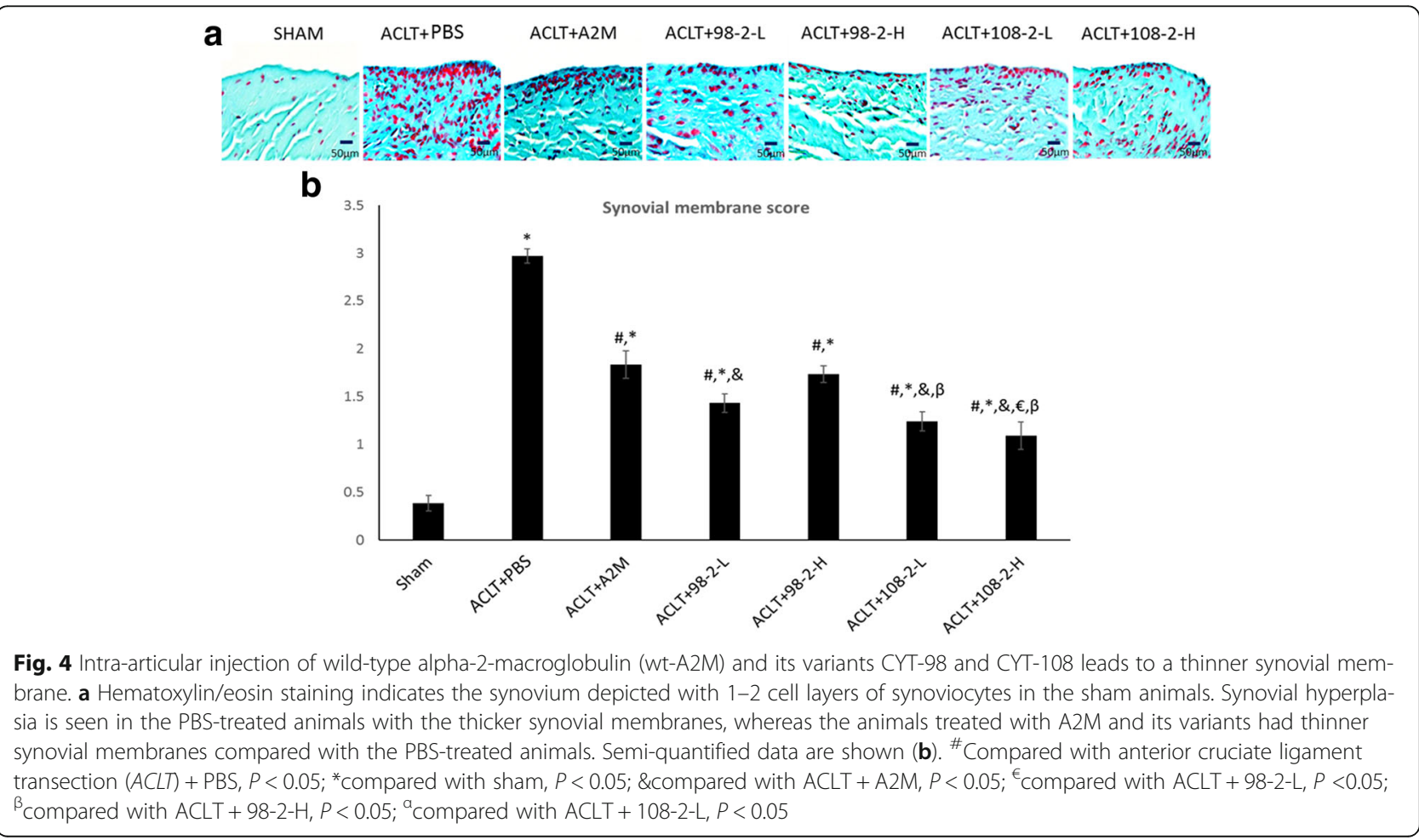


MMP-13 staining

a SHAM ACLT+PBS ACLT+A2M ACLT+98-2-L ACLT+98-2-H ACLT+108-2-L ACLT+108-2-H Antibody control

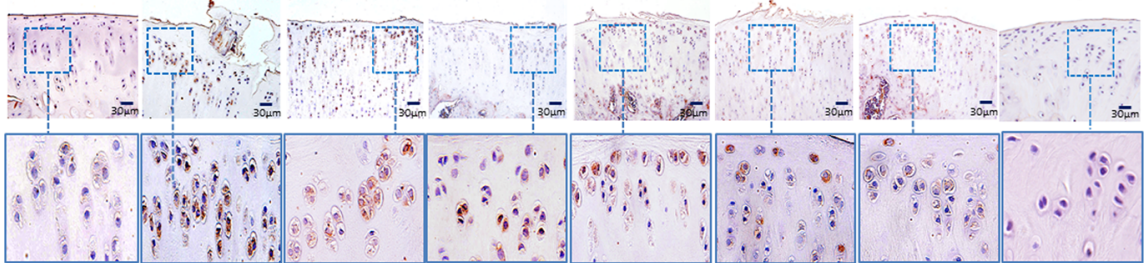

b

Type $X$ collagen staining

D SHAM ACLT+ PBS ACLT+A2M ACLT+98-2-L ACLT+98-2-H ACLT+108-2-L ACLT+108-2-H Antibody control

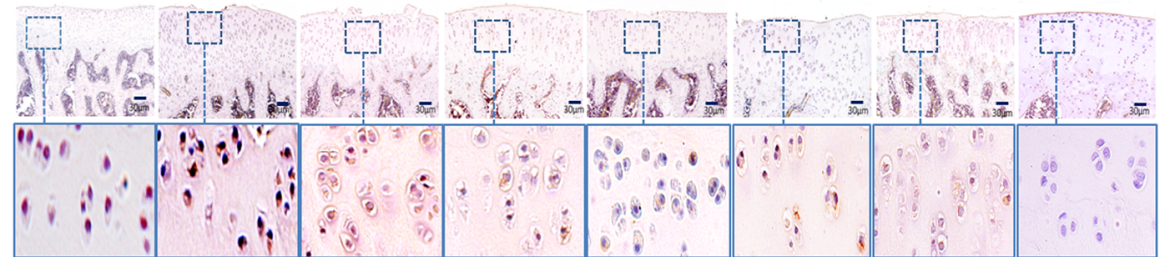

C

Type II collagen degraded products

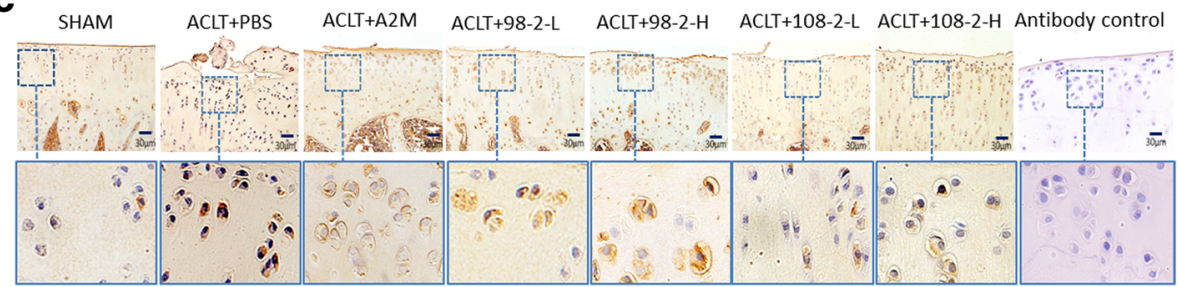

d Type II collagen staining

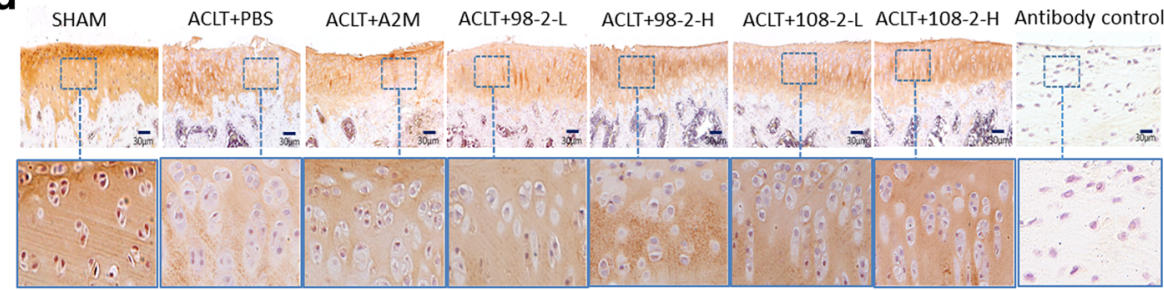

e

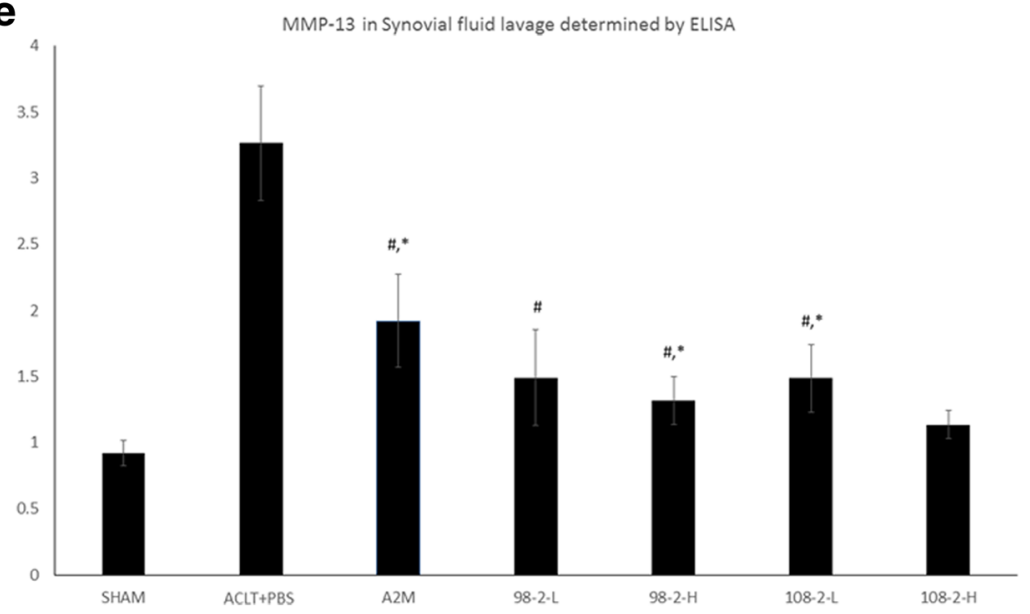

Fig. 5 (See legend on next page.) 
(See figure on previous page.)

Fig. 5 Matrix metalloproteinase 13 (MMP-13) (a), type X collagen (b), and type II degraded products staining (c) were elevated in rats that underwent anterior cruciate ligament transection (ACLT) and PBS treatment, but was lower in the alpha-2-macroglobulin (A2M) and A2M-variants-treated and sham-operated rats, which is consistent with reduced osteoarthritis damage in these rats. In contrast, type II collagen expression in articular cartilage was higher in the A2M and A2M-variants-treated and sham-operated rats than in rats that underwent ACLT and PBS treatment (d). The bottom panels are higher-magnification views of the boxed areas in the top panels. ELISA further confirmed that A2M and its variants partially inhibit MMP-13 (e). In A2M and A2M-variants-treated rats, the concentration of MMP-13 in SF was lower than that in the rats that underwent ACLT and PBS treatment but it was still higher than that in in sham-operated rats. Values are the mean $\pm \mathrm{SD} .{ }^{*}$ Compared with $\mathrm{ACLT}+\mathrm{PBS}, P<0.05 ;{ }^{*} \mathrm{Compared}$ with sham, $P<0.05$; \&compared with ACLT + A2M, $P<0.05 ;{ }^{\epsilon}$ compared with ACLT + 108-2-L, $P<0.05$

2-L, $\mathrm{P}<0.05 ; \beta$ Compared with ACLT $+98-2-\mathrm{H}, \mathrm{P}<0.05$; $\alpha$ Compared with ACLT $+108-2-\mathrm{L}, \mathrm{P}<0.05$.

\section{Discussion}

As A2M inhibits all classes of endoproteases [5, 21, 22], it could be used to mitigate the progression of OA by neutralizing cartilage catabolic factors. Studies have shown that A2M inhibits the activities of ADAMTS-4, ADAMTS-5, ADAMTS-7, and ADAMTS-12 [21, 22], reduces ligament stump resorption following ACL injury [15], and enhances tendon-bone healing of ACL grafts by inhibiting MMP-13 activity [16]. Thus, the balance of the protease/A2M in vivo may play an important role in mediating cartilage destruction by catabolic enzymes. However, A2M is not present in vivo at sufficient levels to counteract the increased concentrations of catabolic factors that appear after joint injury. The level of A2M in serum is $1.53 \mathrm{mg} / \mathrm{ml}$ and the level of A2M in OA SF is $0.24 \mathrm{mg} / \mathrm{ml}$ as compared to MMP-13 expression in serum of $91.1 \mathrm{ng} / \mathrm{ml}$ and MMP-13 expression in SF of $251 \mathrm{ng} / \mathrm{ml}$ [5]. Higher A2M concentration is associated with lower MMP-13 content [5]. The difference is thought to be due to the large molecular weight of A2M, which prevents it's migration from the blood into the SF $[14,15]$. Thus, supplemental A2M may be a potential strategy to attenuate cartilage degeneration by reducing these catabolic enzymes induced by joint injury.

Although autologous A2M is an exciting and potential candidate for $\mathrm{OA}$ treatment, clinicians may find the time, training, expense, and potential safety concerns of drawing blood and preparing autologous $\mathrm{A} 2 \mathrm{M}$ in an outpatient setting burdensome. An off-the-shelf, optimized recombinant form of $\mathrm{A} 2 \mathrm{M}$ and its target variants could eliminate these drawbacks. Our FMT and histology results demonstrated that the recombinant target variants of A2M do attenuate OA damage by inhibiting cartilagedegraded enzymes more effectively than wt-A2M.

FMT is a sensitive bio-imaging method providing noninvasive, deep tissue in vivo imaging and it allows for the evaluation of disease progression at multiple time points without sacrifice of the animal [12, 23-25]. In this study,

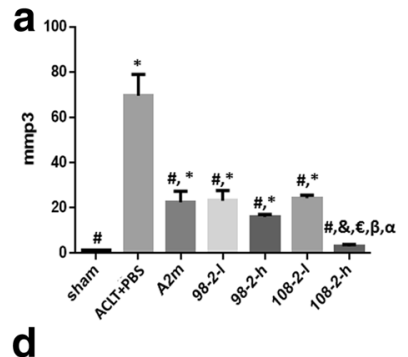

d

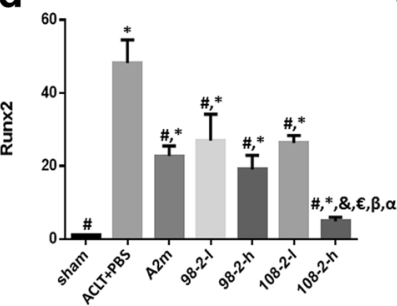

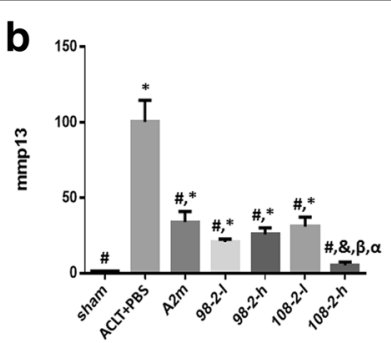

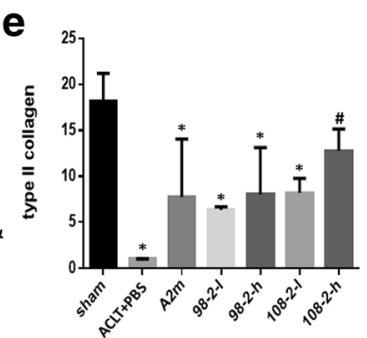

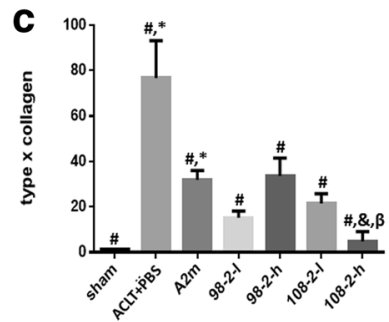

f

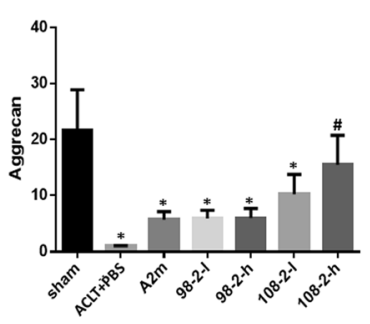

Fig. 6 Supplemental intra-articular wild-type alpha-2-macroglobulin (A2M) and its variants CYT-98 and CYT-108 reduce catabolism and enhance anabolic metabolism in the anterior cruciate ligament transection (ACLT) rat osteoarthritis model. The levels of mRNA of the matrix metalloproteinase (MMP)-3(a), MMP-13(b), type X collagen(c), and Runx2(d) were expressed at a lower level in rats that were administered A2M and its variants as compared to the rats that underwent ACLT and PBS treatment. In contrast, the levels of mRNA for type II collagen (e) and aggrecan (f) followed an opposite pattern. Both of them were increased in rats that were administered A2M and its variants as compared to the rats that underwent ACLT and PBS treatment. Values are the mean \pm SEM. ${ }^{\#}$ Compared with ACLT + PBS, $P<0.05 ;{ }^{*}$ compared with sham group, $P<0.05 ; \&$ compared with ACLT + $\mathrm{A} 2 \mathrm{M}, \mathrm{P}<0.05 ;{ }^{\epsilon}$ compared with ACLT $+98-2-\mathrm{L}, P<0.05 ;{ }^{\beta}$ compared with ACLT $+98-2-\mathrm{H}, P<0.05 ;{ }^{a}$ compared with ACLT $+108-2-\mathrm{L}, P<0.05$ 
we found that $\mathrm{A} 2 \mathrm{M}$ and its variants are able to specifically inhibit proteases and MMPs either in ex vivo cartilage or in vivo ACLT rat OA models immediately after injury compared to PBS-treated animals. The targeted variants of $\mathrm{A} 2 \mathrm{M}$ were more efficacious than wt-A2M in the BCE experiments. A significant decrease of ProSense and MMPSense detected by FMT was observed immediately in the animals treated by $\mathrm{A} 2 \mathrm{M}$ and its variants compared with the animals treated with $\mathrm{PBS}$ in vivo in the ACLT rat OA model. The level of the ProSense remained at a low level at weeks 4 and 6 after surgery even without treatment. This suggests that ProSense is an acute indicator of inflammation and not sensitive for late stages of OA. This finding is consistent with previous reports [5]. Furthermore, the level of MMPSense increased again gradually when A2M was not re-dosed at weeks 4 and 6 . This indicates that MMPs may have two peaks. The first phase appears after the initial trauma to the joint. The second peak is not related to the acute joint injury but is associated with progressive cartilage degeneration. The gradual increase in protease activity suggests to us that a continuing constant supplemental level of A2M may be necessary to prevent catabolic degeneration after joint injury to prevent development of post traumatic OA.

Our histological and biochemistry data further demonstrated that supplemental A2M and its variants not only attenuate cartilage damage and inhibit catabolic factor MMP-13, but also enhance cartilage matrix $\mathrm{Col} 2$ and aggrecan synthesis. The increase in collagen and aggrecan suggests that A2M may have cartilage repair functions or at least does not interfere with cartilage matrix synthesis to proceed. This finding is consistent with a previous report in which a high dose of A2M did not induce chondrocyte death [5]. The findings strongly indicate that $\mathrm{A} 2 \mathrm{M}$ and its variants are promising bioinhibitors for catabolic proteases, and supplemental intra-articular injection of A2M soon after injury may provide a chondral protection effect in vivo in the ACLinjured knee by reducing the presence of local catabolic proteases. Our data also clearly demonstrate these variants of A2M have similar functions compared with wtA2M. Especially, the targeted 108 variant of A2M has demonstrated a stronger chondroprotective effect to prevent or delay cartilage degeneration compared to wtA2M and 98 variant.

Collected evidence has demonstrated that $\mathrm{A} 2 \mathrm{M}$ has the ability to bind cytokines, such as IL-1b and TNFa and that it also enters cells to regulate cellular responses to other growth factors and cytokines [26-28]. The mechanism by which targeted $\mathrm{A} 2 \mathrm{M}$ variants enhance this function is not clear. Since the cytokine binding sites in the A2M variants were not altered, it is very likely these target $\mathrm{A} 2 \mathrm{M}$ variants regulate the process through the inhibition of the catabolic enzymes [5, 21, 23-25], but not through binding to the cytokines or altering their function. Further study to explore the strong inhibitory ability of the designed variants to the cytokines compared with wtA2M is warranted.

One limitation of our study was that the SF samples were obtained from the knees after an injection of $100 \mu \mathrm{l}$ of saline, and cycling and impartial recovery of diluted fluid. Lavage was required to obtain SF from the rat small joint cavity. All SF measurements should be normalized, i.e. using serum and SF urea. Baseline fluid volume, amongst other factors, might have differed in the SF results. Unfortunately, we did not collect blood samples in this study and were unable to collect enough SF samples, due to the small joint volume, in order to normalize the SF results for the urea experiment. Thus, this MMP-13 data analysis does not preclude other variables such as changes in synovial vascular permeability of protein content with OA onset, although no evidence of joint effusion was noted prior to the lavages.

\section{Conclusion}

Our data show that A2M protects cartilage following joint injuries that can progress to OA. Our engineered, recombinant $\mathrm{A} 2 \mathrm{M}$ variants are more protective than the wild-type.

\section{Abbreviations \\ A2M: Alpha-2- macroglobulin; ACAN: Aggrecan; ACL: Anterior cruciate ligament; ACLT: Anterior cruciate ligament transection; ANOVA: analysis of variance; BCE: Bovine articular cartilage explants; CDNA: Complementary DNA; Col: Collagen; Ct: Cycle threshold; DMEM: Dulbecco's modified Eagle's medium; ELISA: Enzyme-linked immunosorbent assay; FMT: Fluorescence molecular tomography; IHC: Immunohistochemical analysis; L: Interleukin; MMP: Matrix metallopeptidase; mRNA: Messenger RNA; OA: Osteoarthritis; OARSI: Osteoarthritis Research Society International; PBS: Phosphate-buffered saline; ROI: Region of interest; SF: Synovial fluid; SGAG: Sulfated glycosaminoglycan; TNF: Tumor necrosis factor; wt: Wild-type}

\section{Acknowledgements}

The authors gratefully acknowledge Dr. Sun, C., Du, G., Wang, S., Chen, C., for help with the surgery and animal care.

\section{Funding}

The project was supported by a grant from NSFC 81572098, 31271033, 81201435, 8160949, SXNSF 20150313012-6, 201605D211024, NIH/NIAMS R01AR059142, and Cytonics Corp. The content is solely the responsibility of the authors and does not necessarily represent the official view of the NIH.

\section{Availability of data and materials}

Not applicable.

\section{Authors' contributions}

YZ participated in the study design, wrote the manuscript, performed most of the experiments, and analyzed data. XCW, SB, GS, and LSH conceived, designed, and manufactured the recombinant A2M and its variants. They also conceived, designed, and performed the bovine articular cartilage explants in vitro study. They also participated in the interpretation of the data and the revision of the manuscript. Cytonics hold all patents related to the composition of A2M variants and its use. LW conceived the in vivo study and participated in its design and data analysis and revised the manuscript with the Cytonics team. All authors have read and approved the final manuscript. 


\section{Competing interests}

The authors declare that they have no competing interests.

\section{Consent for publication}

Not applicable.

\section{Ethics approval and consent to participate}

This study did not need consent from any individuals/patients because no humans were involved in this study. Ethical approval was needed for this study and it was approved by animal welfare committee of Rhode Island Hospital (approved protocol 0011-15).

\section{Disclosures}

YZ, XW, and LW are associated with Brown University. SB, GS, and LH are employees at Cytonics Corporation.

\section{Publisher's Note}

Springer Nature remains neutral with regard to jurisdictional claims in published maps and institutional affiliations.

\section{Author details}

'Department of Orthopedics, the second hospital of the Shanxi Medical University, Taiyuan, China. ${ }^{2}$ Cytonics Corporation, 6917 Vista Pkwy N., Suite 14, West Palm Beach, FL 33411, USA. 'Department of Orthopedics, Alpert Medical School of Brown University/Rhode Island Hospital, Providence, RI, USA

Received: 5 January 2017 Accepted: 6 June 2017

Published online: 25 July 2017

\section{References}

1. Anderson DD, Chubinskaya S, Guilak F, Martin JA, Oegema TR, Olson SA, Buckwalter JA. Post-traumatic osteoarthritis: improved understanding and opportunities for early intervention. J Orthop Res. 2011;29(6):802-9.

2. Kim KS, Choi HM, Lee Y-A, Choi IA, Lee S-H, Hong S-J, Yang H-I, Yoo MC. Expression levels and association of gelatinases MMP-2 and MMP-9 and collagenases MMP-1 and MMP-13 with VEGF in synovial fluid of patients with arthritis. Rheumatol Int. 2011;31(4):543-7.

3. Kanbe K, Takemura T, Takeuchi K, Chen Q, Takagishi K, Inoue K. Synovectomy reduces stromal-cell-derived factor-1 (SDF-1) which is involved in the destruction of cartilage in osteoarthritis and rheumatoid arthritis. J Bone Joint Surg Br. 2004; 86(2):296-300.

4. Wei L, Fleming BC, Sun X, Teeple E, Wu W, Jay GD, Elsaid KA, Luo J, Machan JT, Chen Q. Comparison of differential biomarkers of osteoarthritis with and without posttraumatic injury in the Hartley guinea pig model. J Orthop Res. 2010;28(7):900-6

5. Wang S, Wei X, Zhou J, Zhang J, Li K, Chen Q, Terek R, Fleming BC, Goldring $M B$, Ehrlich MG, et al. Identification of alpha2-macroglobulin as a master inhibitor of cartilage-degrading factors that attenuates the progression of posttraumatic osteoarthritis. Arthritis Rheumatol. 2014;66(7):1843-53.

6. Green DM, Noble PC, Bocell Jr JR, Ahuero JS, Poteet BA, Birdsall HH. Effect of early full weight-bearing after joint injury on inflammation and cartilage degradation. J Bone Joint Surg Am. 2006;88(10):2201-9.

7. Backus JD, Furman BD, Swimmer T, Kent CL, McNulty AL, Defrate LE, Guilak F, Olson SA. Cartilage viability and catabolism in the intact porcine knee following transarticular impact loading with and without articular fracture. J Orthop Res. 2011;29(4):501-10.

8. Borrelli Jr J, Tinsley K, Ricci WM, Burns M, Karl IE, Hotchkiss R. Induction of chondrocyte apoptosis following impact load. J Orthop Trauma. 2003;17(9): 635-41.

9. Tochigi Y, Buckwalter JA, Martin JA, Hillis SL, Zhang P, Vaseenon T, Lehman $A D$, Brown TD. Distribution and progression of chondrocyte damage in a whole-organ model of human ankle intra-articular fracture. J Bone Joint Surg Am. 2011:93(6):533-9.

10. Wei F, Zhou J, Wei X, Zhang J, Fleming BC, Terek R, Pei M, Chen Q, Liu T, Wei L. Activation of Indian hedgehog promotes chondrocyte hypertrophy and upregulation of MMP-13 in human osteoarthritic cartilage. Osteoarthr Cartil. 2012;20(7):755-63.

11. Wei FMD, Li Y, Zhang G, Wei X, Lee JK, Wei L. Attenuation of osteoarthritis via blockade of the SDF-1/CXCR4 signaling pathway. Arthritis Res Ther 2012;14(4):R177. [Epub ahead of print](PMCID:PMC3580571).
12. Zhou J, Chen Q, Lanske B, Fleming BC, Terek R, Wei X, Zhang G, Wang S, Li $\mathrm{K}$, Wei L. Disrupting the Indian hedgehog signaling pathway in vivo attenuates surgically induced osteoarthritis progression in Col2a1-CreERT2; Ihhfl/fl mice. Arthritis Res Ther. 2014;16(1):R11.

13. Jay GD, Elsaid KA, Kelly KA, Anderson SC, Zhang L, Teeple E, Waller K, Fleming BC. Prevention of cartilage degeneration and gait asymmetry by lubricin tribosupplementation in the rat following anterior cruciate ligament transection. Arthritis Rheum. 2012;64(4):1162-71.

14. Salvesen G, Enghild JJ. alpha-Macroglobulins: detection and characterization. Methods Enzymol. 1993;223:121-41.

15. Demirag B, Sarisozen B, Durak K, Bilgen OF, Ozturk C. The effect of alpha-2 macroglobulin on the healing of ruptured anterior cruciate ligament in rabbits. Connect Tissue Res. 2004;45(1):23-7.

16. Demirag B, Sarisozen B, Ozer O, Kaplan T, Ozturk C. Enhancement of tendon-bone healing of anterior cruciate ligament grafts by blockage of matrix metalloproteinases. J Bone Joint Surg Am. 2005;87(11):2401-10.

17. Du G, Zhan H, Ding D, Wang S, Wei X, Wei F, Zhang J, Bilgen B, Reginato AM, Fleming BC, et al. Abnormal mechanical loading induces cartilage degeneration by accelerating meniscus hypertrophy and mineralization after ACL injuries in vivo. Am J Sports Med. 2016;44(3):652-63.

18. Jay GD, Fleming BC, Watkins BA, MCHugh KA, Anderson SC, Zhang LX, Teeple E, Waller KA, Elsaid KA. Prevention of cartilage degeneration and restoration of chondroprotection by lubricin tribosupplementation in the rat following anterior cruciate ligament transection. Arthritis Rheum. 2010;62(8): 2382-91.

19. Pritzker KPH, Gay S, Jimenez SA, Ostergaard K, Pelletier JP, Revell PA, Salter D, van den Berg WB. Osteoarthritis cartilage histopathology: grading and staging. Osteoarthr Cartil. 2006;14(1):13-29.

20. Gerwin N, Bendele AM, Glasson S, Carlson CS. The OARSI histopathology initiative - recommendations for histological assessments of osteoarthritis in the rat. Osteoarthr Cartil. 2010;18 Suppl 3:S24-34.

21. Tortorella MD, Arner EC, Hills R, Easton A, Korte-Sarfaty J, Fok K, Wittwer AJ, Liu R-Q, Malfait A-M. Alpha2-macroglobulin is a novel substrate for ADAMTS-4 and ADAMTS-5 and represents an endogenous inhibitor of these enzymes. Jiol Chem. 2004:279(17):17554-61.

22. Luan Y, Kong L, Howell DR, llalov K, Fajardo M, Bai XH, Di Cesare PE, Goldring MB, Abramson SB, Liu CJ. Inhibition of ADAMTS-7 and ADAMTS-12 degradation of cartilage oligomeric matrix protein by alpha-2-macroglobulin. Osteoarthr Cartil. 2008;16(11):1413-20.

23. Abbink JJ, Kamp AM, Nieuwenhuys EJ, Nuijens JH, Swaak AJ, Hack CE. Predominant role of neutrophils in the inactivation of alpha 2-macroglobulin in arthritic joints. Arthritis Rheum. 1991:34(9):1139-50.

24. Zhang L, Yang M, Yang D, Cavey G, Davidson P, Gibson G. Molecular interactions of MMP-13 C-terminal domain with chondrocyte proteins. Connect Tissue Res. 2010;51(3):230-9.

25. Abbink JJ, Nuijens JH, Eerenberg AJ, Huijbregts CC, van Schijndel RJS, Thijs LG, Hack CE. Quantification of functional and inactivated alpha 2-macroglobulin in sepsis. Thromb Haemost. 1991:65(1):32-9.

26. LaMarre J, Wollenberg GK, Gonias SL, Hayes MA. Cytokine binding and clearance properties of proteinase-activated alpha 2-macroglobulins. Lab Investig. 1991;65(1):3-14

27. Wollenberg GK, LaMarre J, Rosendal S, Gonias SL, Hayes MA. Binding of tumor necrosis factor alpha to activated forms of human plasma alpha 2 macroglobulin. Am J Pathol. 1991;138(2):265-72.

28. Borth W, Scheer B, Urbansky A, Luger TA, Sottrup-Jensen L. Binding of IL-1 beta to alpha-macroglobulins and release by thioredoxin. J Immunol. 1990; 145(11):3747-54. 